\title{
Ultrafast modulation of electronic structure by coherent phonon excitations
}

\author{
J. Weisshaupt ${ }^{1}$ A. Rouzée, ${ }^{1}$ M. Woerner,,${ }^{1, *}$ M. J. J. Vrakking, ${ }^{1}$ T. Elsaesser, ${ }^{1}$ E. L. Shirley, ${ }^{2}$ and A. Borgschulte ${ }^{3}$ \\ ${ }^{1}$ Max-Born-Institut für Nichtlineare Optik und Kurzzeitspektroskopie, D-12489 Berlin, Germany \\ ${ }^{2}$ National Institute of Standards and Technology, Gaithersburg, Maryland 20899-8441, USA \\ ${ }^{3}$ Swiss Federal Laboratories for Materials Science and Technology, Laboratory for Advanced Analytical Technologies, \\ EMPA, CH-8600 Dübendorf, Switzerland
}

(Received 24 October 2016; published 1 February 2017)

\begin{abstract}
Femtosecond x-ray absorption spectroscopy with a laser-driven high-harmonic source is used to map ultrafast changes of x-ray absorption by femtometer-scale coherent phonon displacements. In $\mathrm{LiBH}_{4}$, displacements along an $A_{g}$ phonon mode at $10 \mathrm{THz}$ are induced by impulsive Raman excitation and give rise to oscillatory changes of x-ray absorption at the Li $K$ edge. Electron density maps from femtosecond x-ray diffraction data show that the electric field of the pump pulse induces a charge transfer from the $\mathrm{BH}_{4}{ }^{-}$to neighboring $\mathrm{Li}^{+}$ions, resulting in a differential Coulomb force that drives lattice vibrations in this virtual transition state.
\end{abstract}

DOI: 10.1103/PhysRevB.95.081101

The complex line shape of x-ray absorption above an edge, the so-called x-ray near-edge absorption structure (XANES), has been described in a multiple scattering picture [1,2] where the photoexcited electron probes the nearest neighbors of the excited atom and quantum interference of the different scattering pathways leads to resonances in the spectral envelope. Changes of interatomic distances result in phase changes of the multiple possible quantum pathways that an excited electron can follow $[3,4]$. This allows for deriving the local atomic structure from the $\mathrm{x}$-ray absorption spectrum. Under stationary conditions this also gives element-specific insight into equilibrium geometries, the electronic structure, and the chemical binding of crystalline and noncrystalline materials [5-9]. An equally valid picture in the near-edge region connects the absorption spectrum with the corehole-perturbed, dipole-transition-accessible local density of states $[10,11]$.

Transient x-ray absorption spectra have been recorded with atto- to picosecond time resolution and provide insight into the electronic excitations and/or chemical processes in atoms, molecular systems, and solids [12-23]. Much less explored is the interplay in real time of atomic displacements and $\mathrm{x}$-ray absorption [22]. In crystalline solids, phonon excitations are characterized by energies below $500 \mathrm{meV}$, femtosecond vibrational periods, and spatial elongations of typically less than a few percent of the interatomic distances. The latter are much too small to be extracted from steady-state $\mathrm{x}$-ray absorption spectra. In the transient regime, optical pulses of a duration shorter than the vibrational period allow for the impulsive excitation of phonon wave packets, i.e., nonstationary superpositions of phonon states which manifest themselves in coherent oscillations along one or several vibrational coordinates. Here, we demonstrate that such coherent motions modulate the electronic structure, and hence the $\mathrm{x}$-ray absorption in the XANES region, even though the displacements are on the femtometer scale. With the help of transient electron density maps determined by femtosecond $\mathrm{x}$-ray diffraction [24], we identify the role of electronic charge rearrangement in the nonresonant Raman excitation process of

\footnotetext{
*woerner@mbi-berlin.de
}

coherent phonons. Detailed information on the Coulomb force field in the "virtual electronic state" of the Raman scattering process is derived in this way.

The experiments presented in the following are based on a femtosecond pump-probe scheme wherein a femtosecond tabletop laser system provides excitation pulses around $800 \mathrm{~nm}$ and generates synchronized soft $\mathrm{x}$-ray probe pulses [25], allowing for transient $\mathrm{x}$-ray absorption spectroscopy with a sub-50 fs time resolution. The experiments are complemented by a theoretical model for the transient x-ray absorption which is based on the Bethe-Salpeter-equation (BSE) approach. Both experimental and theoretical details are given in the Supplemental Material (SM) [26]. Finally, we combine information from recent femtosecond $\mathrm{X}$-ray diffraction experiments [24] with that from the present experiment to draw a complete microscopic picture of the Raman process.

Crystalline $\mathrm{LiBH}_{4}$ represents a most interesting compound for a combined femtosecond $\mathrm{x}$-ray absorption and diffraction study since it contains only light elements and thus makes the quasi-instantaneous response of valence electrons accessible in experiment. In the femtosecond x-ray diffraction experiment, the intensity changes $\Delta I_{h k l} / I_{h k l}^{0}$ of Bragg peaks are dominated by changes of the valence electron density. Moreover, Li shows its $K$ edge in a spectral range of high-harmonic generation conveniently accessible with high repetition laser drivers. From a more general point of view, $\mathrm{LiBH}_{4}$ is a potential hydrogen storage material with high Li-ion conductivity and has been extensively studied for that reason [27].

In our experiments, we study a polycrystalline film of lithium borohydride $\left(\mathrm{LiBH}_{4}\right)$ [Fig. 1(a)]. The film has a thickness of approximately $1 \mu \mathrm{m}$ and is deposited on a $50 \mathrm{~nm}$ thick diamond substrate. Steady-state and transient $\mathrm{x}$-ray absorption spectra are recorded in transmission around the $\mathrm{Li}$ $K$ edge at $60 \mathrm{eV}$. The stationary x-ray absorption spectrum [Fig. 2(a)] was measured with the femtosecond two-color high-harmonic (HH) source driven by 800 and $1300 \mathrm{~nm}$ pulses (black line, spectral resolution $200 \mathrm{meV}$ ). In contrast to single-color $\mathrm{HHG}$, the use of a two-color driving field for the HHG process allows us to generate a continuous soft X-ray spectrum near the Li $K$ edge [25]. The x-ray absorption spectrum displays a pronounced excitonic feature at the $\mathrm{Li} K$ edge near $60 \mathrm{eV}$ which is due to the mutual attraction of the 

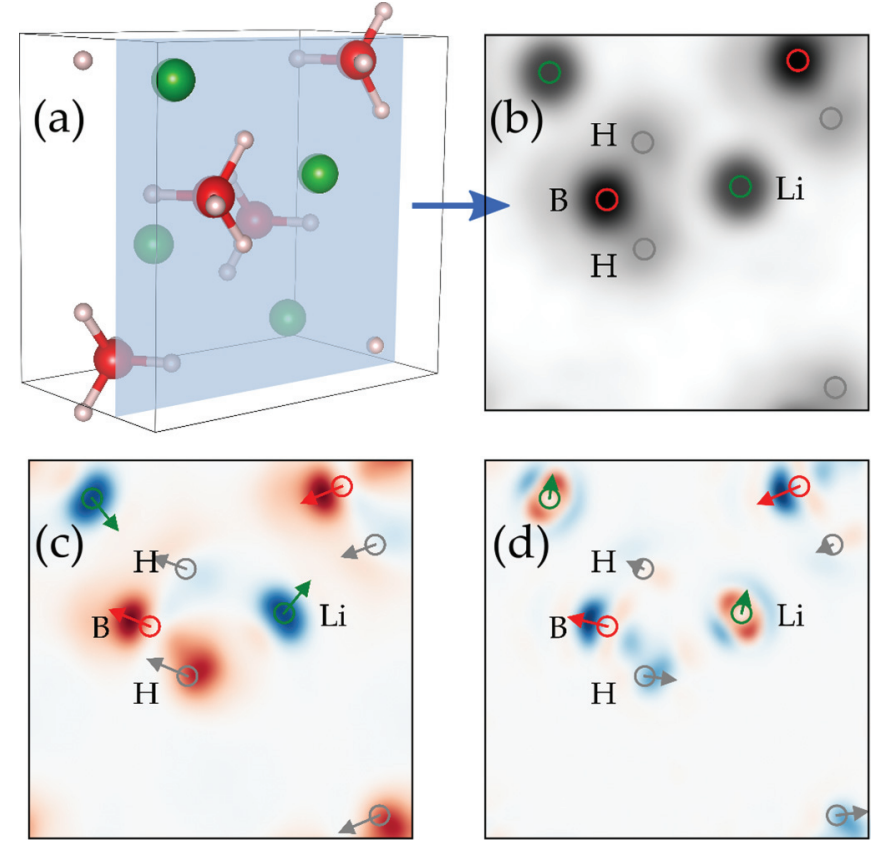

FIG. 1. (a) Unit cell of lithium borohydride with the $\mathrm{Li}^{+}$(green) and $\mathrm{BH}_{4}{ }^{-}$ions (B: red; $\mathrm{H}$ : gray). The shaded area marks the $y=\frac{1}{4}$ plane. (b) Equilibrium electron density $\rho_{0}(\mathbf{r})$ in the $y=\frac{1}{4}$ plane. (c) Transient differential electronic charge density $\Delta \rho(\mathbf{r}, t=0)$ reported in Ref. [24]. Red and blue coloration indicate negative and positive changes in the local electron density. The arrows indicate the impulsively excited $10 \mathrm{THz} A_{g}$ Raman mode. (d) Differential Coulomb potential $V_{\text {diff }}(\mathbf{r}, 0)$ generated from the transient differential electron density in (c). The arrows indicate the electric field $\nabla V_{\text {diff }}(\mathbf{r}, 0)$. The boron nuclei are strongly accelerated by the impulsive force of the induced electronic polarization due to the strong overlap between the $A_{g}$ mode's elongations and the impulsive force.

excited electron and the core hole, and a slightly modulated plateaulike XANES region above the $K$ edge. The theoretical spectrum (blue line) calculated from the BSE formalism (see $\mathrm{SM}$ ) reproduces the main features of the measured spectrum.

$\mathrm{LiBH}_{4}$ exhibits a rich phonon spectrum due to the large unit cell comprising 24 atoms [Fig. 1(a)]. In the pump-probe experiments, a $50 \mathrm{fs}$ laser pulse centered at $800 \mathrm{~nm}$ (photon energy $1.5 \mathrm{eV}$ ) excites coherent phonons via a nonresonant impulsive Raman process [28]. In this scheme, Raman-active modes at frequencies within the bandwidth of the femtosecond pulse are excited, in particular, the $A_{g}$ mode at $v=10 \mathrm{THz}$ $\left(300 \mathrm{~cm}^{-1}\right)$. The pump pulse at $1.5 \mathrm{eV}$ is far below the $\approx 7 \mathrm{eV}$ band gap of $\mathrm{LiBH}_{4}$. Multiphoton absorption of pump light is negligible for the present peak intensity of $I_{\text {pump }} \approx$ $10^{11} \mathrm{~W} / \mathrm{cm}^{2}$, which corresponds to an electric field amplitude of about $10^{7} \mathrm{~V} / \mathrm{cm}$. The electric field, however, induces a relocation of electronic charge from the electron-rich $\mathrm{BH}_{4}{ }^{-}$ ion to the neighboring $\mathrm{Li}^{+}$ion in a lithium borohydride ion pair [cf. Fig. 1(a)], as previously observed in femtosecond $\mathrm{X}$-ray diffraction experiments performed with similar pump pulses [24].

Upon impulsive phonon excitation, oscillatory absorption changes are observed in the plateaulike XANES region.

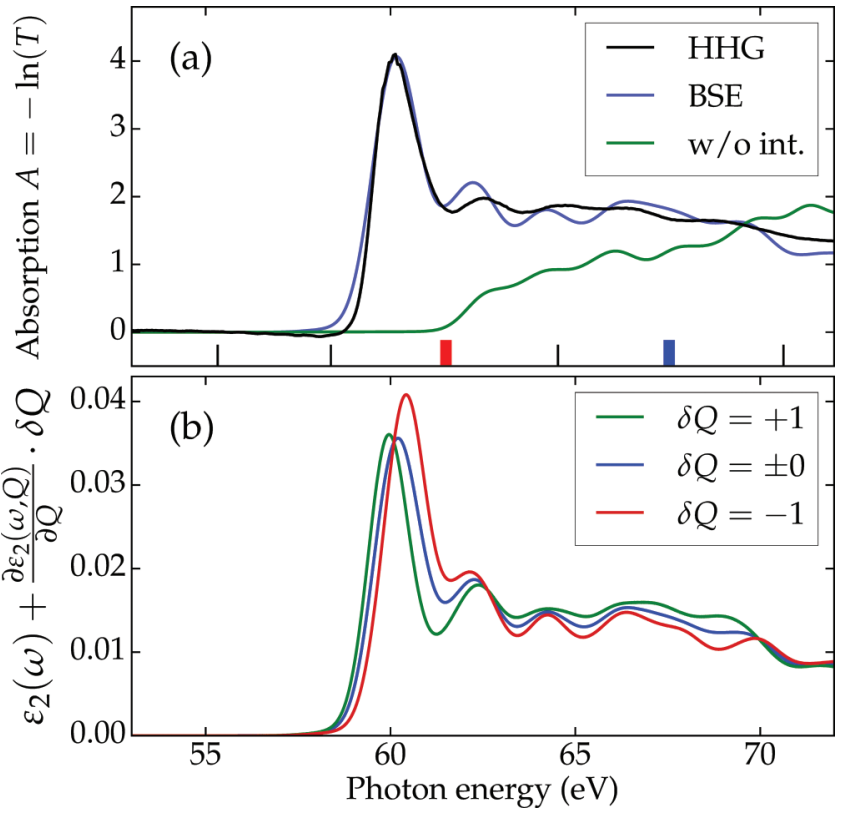

FIG. 2. (a) Black: Linear absorption spectrum of $\mathrm{LiBH}_{4}$ at the Li $K$ edge as measured with a two-color high-harmonic (HHG) source. Blue: Theoretical spectrum calculated using a Bethe-Salpeter approach. A transition into a bound excitonic state is observed at $60 \mathrm{eV}$, followed by transitions into free conduction band states at higher energies. The oscillations above $62 \mathrm{eV}$ are due to band structure effects. A calculation without electron-hole interaction (green line) does not account for the experimental spectrum but does show the dipole-matrix-element-allowed density of states. Tick marks indicate the positions of high harmonics generated using an $800 \mathrm{~nm}$ laser used in transient absorption measurements shown in Figs. 3(a)-3(c). (b) Theoretical x-ray absorption spectra calculated for strongly exaggerated elongation amplitudes of the $10 \mathrm{THz} A_{g}$ phonon mode in units of $[\delta Q]=\AA \sqrt{\mathrm{amu}}$. Pronounced absorption changes occur both in the excitonic and plateau regions of the spectral envelope.

In Figs. 3(a) and 3(b), the change of absorption $\Delta A=$ $-\ln \left(T / T_{0}\right)$ at photon energies of $61.5 \mathrm{eV}$ [Fig. 3(a)] and $67 \mathrm{eV}$ [Fig. 3(b)] is plotted as a function of pump-probe delay $\left(T, T_{0}\right.$ being the sample transmission with and without excitation). The transients were measured on individual high harmonics [cf. red and blue bars at the bottom of Fig. 2(a)] generated with the $800 \mathrm{~nm}$ laser pulses.

At both spectral positions, the absorption change shows a maximum amplitude of approximately $\Delta A=2 \times 10^{-4}$ and an oscillatory time evolution with a period of $100 \mathrm{fs}$. There is no detectable nonlinear signal below the Li $K$ edge. The Fourier spectrum of the oscillations in Fig. 3(c) (bottom) consists of a predominant feature around $10 \mathrm{THz}$, the frequency of the $A_{g}$ phonon of $\mathrm{LiBH}_{4}$. For comparison, we plot a high-resolution Raman spectrum of $\mathrm{LiBH}_{4}$ in the upper part of Fig. 3(c) (cf. SM).

We also recorded transient $\mathrm{x}$-ray absorption spectra with the continuous two-color high-harmonics probe [25] which are presented in Fig. 3(d). Due to the larger fluctuations of the two-color HHG source (for details, see SM) we applied a higher pump field in this experiment and investigated a somewhat thicker sample resulting together in a five times 


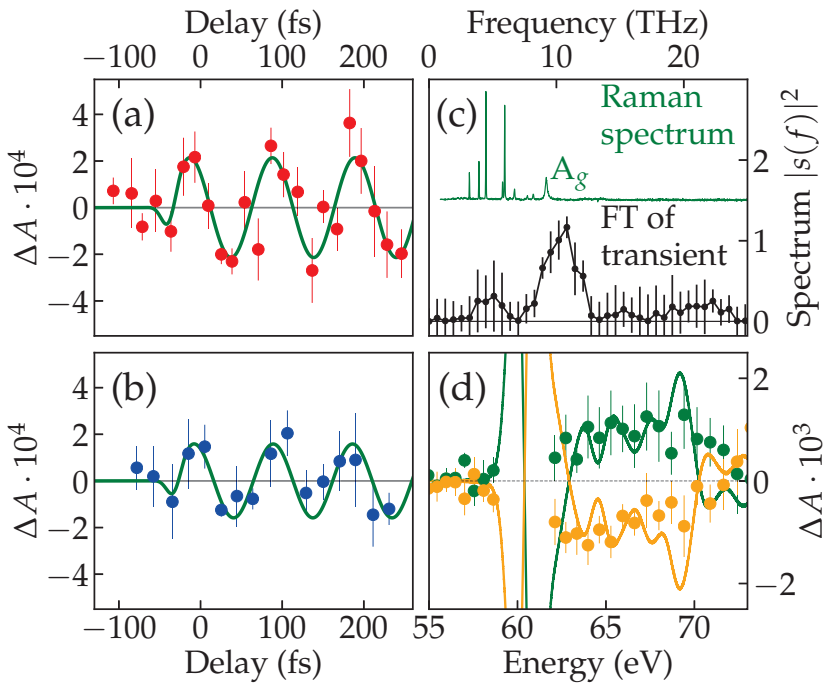

FIG. 3. Transient change of x-ray absorption at (a) $61.5 \mathrm{eV}$ and (b) $67.5 \mathrm{eV}$, the photon energies of individual harmonics. The change of absorption $\Delta A=-\ln \left(T / T_{0}\right)$ is plotted as a function of pump-probe delay ( $T$ and $T_{0}$ are the sample transmission with and without excitation) with $1 \sigma$ error bars. Both transients display strong oscillations due to impulsively excited coherent phonons with a main frequency around $10 \mathrm{THz}$ (solid lines). (c) Bottom: Fourier transform of the oscillations in (a). Top: Spontaneous Raman spectrum of $\mathrm{LiBH}_{4}$. (d) Transient absorption spectra measured with the two-color HHG source [symbols, note that the signal is higher than in (a) and (b) due to different experimental parameters] derived by averaging the absorption changes at the maxima and minima of oscillatory pump-probe traces. The solid lines are derived from the calculated spectra in Fig. 2(b) by rescaling the phonon elongations $\delta Q$ to the values estimated from the experiments. The high optical density of the film sample did not allow for extending the measurements into the near-edge region of the spectrum, which features the excitonic peak.

larger nonlinear signal compared to Figs. 3(a) and 3(b). To reduce the error bars, we averaged the intensity changes at the maxima (green symbols) and minima (orange symbols) of the oscillatory pump probe traces up to a maximum delay time of $500 \mathrm{fs}$. In the range of the excitonic resonance between 59 and $62 \mathrm{eV}$, the optical density of the $\mathrm{LiBH}_{4}$ film was too high to measure reliable transients. The solid lines in Fig. 3(d) were calculated using the BSE approach and will be discussed below.

Our experimental results provide direct evidence for a modulation of the $\mathrm{x}$-ray absorption by coherent phonon motions in the $\mathrm{LiBH}_{4}$ crystallites. We first discuss how such motions are generated, followed by an in-depth analysis of the relevant Raman polarizability in the presence of the strong electric field of the pump pulse. In an impulsive nonresonant Raman excitation process, the optical pump pulse exerts an effective force on a particular phonon mode $Q_{m}$ which is given by

$$
F\left(Q_{m}, t\right)=\frac{1}{8 \pi} \mathbf{E}(t) \cdot \frac{\partial \boldsymbol{\alpha}\left(Q_{m}\right)}{\partial Q_{m}} \cdot \mathbf{E}(t) .
$$

Here, $Q_{m}$ represents the phonon (normal) coordinate, $\boldsymbol{\alpha}\left(Q_{m}\right)$ the polarizability tensor of the material, and $\mathbf{E}(t)$ the pump field at the wave vector $\mathbf{q}=0$ as described in Ref. [28].
The force is proportional to the square of the electric field, reflecting the fact that Raman scattering is second order in the external field. In impulsive Raman scattering, all modes with frequencies within the spectral bandwidth of the driving field are excited. The displacement along the mode $Q_{m}$ is given by

$$
\delta Q=\frac{1}{8 \pi \omega_{m}} \sum_{i, j} \frac{\partial \alpha_{i j}}{\partial Q_{m}}\left|\int_{-\infty}^{\infty} E_{i}(\tau) E_{j}(\tau) e^{i \omega_{m} \tau} d \tau\right|,
$$

where $\omega_{m}$ is the vibrational frequency. The individual atomic displacements $\Delta \mathbf{r}$ are derived from $\delta Q$ by $\Delta \mathbf{r}=\delta Q \mathbf{M}^{-\frac{1}{2}}$. $\mathbf{Q}_{m} / Q_{m}$, with the Raman mode eigenvector $\mathbf{Q}_{m} / Q_{m}$ and the mass matrix $\mathbf{M}$. We performed a measurement of the absolute Raman polarizability for the $10 \mathrm{THz} A_{g}$ mode to estimate the actual displacements under our excitation conditions (cf. $\mathrm{SM})$. For the electric field in the femtosecond experiment of $10^{7} \mathrm{~V} / \mathrm{cm}$, one estimates $\delta Q \approx 3 \times 10^{-4} \AA \mathrm{amu}^{0.5}$ and a displacement of the $\mathrm{Li}$ atoms of $3 \times 10^{-5} \AA$. It is important to note that the atomic displacements induced by impulsive Raman excitation are much smaller than for displacively excited phonons and far below the detection limit of time-resolved x-ray diffraction [29].

The thin $\mathrm{LiBH}_{4}$ film studied here is a macroscopic disordered ensemble of crystallites. This results in spatial averaging of the (nonlinear) x-ray absorption spectrum over polarization direction with respect to local crystallographic axes. The Raman polarizability tensor can be expressed as a Taylor series with respect to $Q_{m}$ :

$$
\alpha_{i j}\left(\omega, Q_{m}\right)=\alpha_{i j}^{0}(\omega)+\frac{\partial \alpha_{i j}}{\partial Q_{m}} Q_{m}+\frac{1}{2} \frac{\partial^{2} \alpha_{i j}}{\partial Q_{m}^{2}} Q_{m}^{2} .
$$

In general, all terms are tensors with symmetry properties depending on the space group of the material and the symmetry of the phonon mode. Considering an individual crystallite, the entire process, i.e., coherent Raman excitation followed by $\mathrm{x}$-ray probing, allows for signal contributions from all Raman active modes. In the averaging process over all crystallites in the macroscopic sample, Raman modes with $A_{g}$ symmetry play a dominant role because the first-order effects of $\partial \alpha_{i j} / \partial Q_{m}$ are observable at the fundamental vibrational frequency. Second-order Raman modes only appear at even harmonics. For the present small vibrational displacements, these contributions are negligible.

The femtometer-scale atomic displacements modify the $\mathrm{X}$-ray absorption. Our analysis is based on the detailed band structure of the periodic crystalline solid, in contrast to an electron scattering picture. Beyond that, our solution of the BSE accounts for many correlation effects and the screened Coulomb interaction between electrons and holes, unlike an independent-particle picture. X-ray absorption spectra are calculated by solving the BSE equation accounting for electric-dipole transitions into all unoccupied band states. For $\mathrm{x}$-ray absorption due to the excitation of core electrons, the core states exhibit negligible band dispersion. As a result, a rearrangement of the nuclei within the unit cell (of constant shape and size) by coherent phonon excitations leaves the core states essentially unchanged but modifies the excited electronic band states and the associated dipole-transition matrix elements, so that nuclear motions are evident in $\mathrm{X}$-ray absorption. Beyond such single-particle properties, the 
electron-core hole attraction also has a strong influence on the x-ray absorption spectrum at the $K$ edge. For a quantitative description, we consider the imaginary part of the dielectric tensor of $\mathrm{LiBH}_{4}, \operatorname{Im} \epsilon_{i j}\left(\omega_{\mathrm{XUV}}, Q_{m}\right)$, which depends on the (soft) X-ray photon energy $\hbar \omega_{\mathrm{XUV}}$. This tensor and its expansion with respect to $Q_{m}$ have the same symmetry properties as $\alpha_{i j}\left(Q_{m}\right)$ [cf. Eq. (3)]. In Sec. IX B 4 of the SM we discuss the relation between theoretically calculated $\epsilon_{2}\left(\omega_{\mathrm{XUV}}, Q_{m}\right)$ [Fig. 2(b)] and the experimentally observed absorption $A\left(\omega_{\mathrm{XUV}}, Q_{m}\right)$ [Fig. 2(a)].

In Fig. 2(b), we present X-ray absorption spectra calculated from the BSE treatment (cf. SM) for different amplitudes of the $10 \mathrm{THz} A_{g}$ mode. This result reveals pronounced changes of the absorption line shape in both the excitonic and the XANES region. For a direct comparison of the theory results with the measured transient spectra in Fig. 3(d), we use a sample thickness of $L \approx 1 \mu \mathrm{m}$ and derive an absolute value of $\delta Q=3 \times 10^{-4} \AA \mathrm{amu}^{0.5}$, i.e., much smaller than the values used to prepare Fig. 2(b), in agreement with the phonon displacements estimated from the measured absolute Raman polarizability [cf. Eq. (2) and SM]. The calculated spectra [solid lines in Fig. 3(d)] are in good agreement with the experiment (symbols), supporting the conclusion that the impulsively excited oscillations of the $A_{g}$ phonon at $10 \mathrm{THz}$ underlie the observed modulation of x-ray absorption, and demonstrating the high sensitivity of $\mathrm{x}$-ray absorption spectra to small atomic displacements.

We now develop a microscopic picture of the impulsive Raman excitation process. The electric field of the pump pulse of approximately $10^{7} \mathrm{~V} / \mathrm{cm}$ modifies the local intra- and interionic potentials. As a result, the electron wave functions become superpositions of electronic states in the valence and conduction band manifolds of $\mathrm{LiBH}_{4}$. The equilibrium spatial distribution of electronic charge $\rho_{0}(\mathbf{r})$ which is shown for the $y=\frac{1}{4}$ plane in Fig. 1(b) is strongly modified by this field-induced coupling of states. Recent femtosecond $\mathrm{x}$-ray diffraction experiments have provided transient differential electron density maps $\Delta \rho(\mathbf{r}, t)$ [24], giving evidence of a laser-induced shift of electronic charge from the electron-rich $\mathrm{BH}_{4}{ }^{-}$ions to neighboring $\mathrm{Li}^{+}$ions [Fig. 1(c)]. This charge transfer is connected with a transient change of the vibrational potentials and results in an effective force displacing the nuclei from their equilibrium positions. In Fig. 1(d), we plot the differential Coulomb potential $V_{\text {diff }}(\mathbf{r}, t=0)$ originating from the electron transfer which characterizes the virtual intermediate state of the Raman process.

The gradient of this potential $\nabla V_{\text {diff }}(\mathbf{r}, 0)$ defines the forces acting on the $\mathrm{BH}_{4}{ }^{-}$and $\mathrm{Li}^{+}$ions [arrows in Fig. 1(d)]. The boron nuclei are strongly accelerated by the impulsive force of the induced electronic polarization. The large overlap between force vectors [Fig. 1(d)] and the normal mode [Fig. 1(c)] favors a strong coherent excitation of the $10 \mathrm{THz} A_{g}$ Raman mode, which is observed in the time-resolved x-ray absorption experiment. Combining the femtosecond diffraction study [24] with the present $\mathrm{X}$-ray absorption experiment gives a complete picture of the Raman process. On the one hand, timeresolved $\mathrm{x}$-ray diffraction uncovers the electronic response to a nonresonant driving field in a spatially resolved way and, in turn, the resulting forces on the ions corresponding to the virtual state in the Raman scattering language. On the other hand, femtosecond $\mathrm{x}$-ray absorption spectroscopy provides information on the motion and displacements of specific nuclei via their transiently modified $K$-edge absorption spectra with a sensitivity down to $\triangle A=10^{-4}$.

In conclusion, ultrafast oscillatory changes of x-ray absorption in $\mathrm{LiBH}_{4}$ have been observed by ultrafast $\mathrm{x}$-ray absorption spectroscopy, based on femtosecond optical pump pulses and synchronized soft x-ray pulses from a laser-driven high-harmonic source. The modulation of the x-ray absorption in the range of the Li $K$ edge is due to impulsive excitation of coherent motions of the $10 \mathrm{THz} A_{g}$ phonon, favored by the relocation of electronic charge in the presence of the pump field. Our results establish ultrafast transient x-ray absorption as a very sensitive probe of phonon quantum coherences and their impact on electronic structures, phenomena relevant for a broad range of functional materials and soft matter samples.

We acknowledge financial support by the Leibniz Gemeinschaft (SAW-2016-MBI-1). The two-color generation scheme was investigated in a collaborative effort with colleagues from the University of Szeged, supported by the National Research, Development and Innovation Office (OTKA project NN107235).
[1] P. A. Lee and J. B. Pendry, Theory of the extended x-ray absorption fine structure, Phys. Rev. B 11, 2795 (1975).

[2] S. I. Zabinsky, J. J. Rehr, A. Ankudinov, R. C. Albers, and M. J. Eller, Multiple-scattering calculations of X-ray absorption spectra, Phys. Rev. B 52, 2995 (1995).

[3] H. W. Sheng, W. K. Luo, F. M. Alamgir, J. M. Bai, and E. Ma, Atomic packing and short-to-medium-range order in metallic glasses, Nature (London) 439, 419 (2006).

[4] I. V. Tomov, D. A. Oulianov, P. Chen, and P. M. Rentzepis, Ultrafast time-resolved transient structures of solids and liquids studied by means of $\mathrm{x}$-ray diffraction and EXAFS, J. Phys. Chem. B 103, 7081 (1999).

[5] J. J. Rehr, R. C. Albers, and S. I. Zabinsky, High-Order MultipleScattering Calculations of X-Ray Absorption Fine Structure, Phys. Rev. Lett. 69, 3397 (1992).
[6] P. A. O’Day, J. J. Rehr, S. I. Zabinsky, and G. E. Brown, Jr., Extended x-ray absorption fine structure (EXAFS) analysis of disorder and multiple scattering in complex crystalline solids, J. Am. Chem. Soc. 116, 2938 (1994).

[7] J. Mustre, Y. Yacoby, E. A. Stern, and J. J. Rehr, Analysis of experimental extended $\mathrm{x}$-ray absorption fine-structure (EXAFS) data using calculated curved-wave, multiple scattering EXAFS spectra, Phys. Rev. B 42, 10843 (1990).

[8] V. Lordi, V. Gambin, S. Friedrich, T. Funk, T. Takizawa, K. Uno, and J. S. Harris, Nearest-Neighbor Configuration on (GaIn)(NAs) Probed by X-Ray Absorption Spectroscopy, Phys. Rev. Lett. 90, 145505 (2003).

[9] A. V. Kolobov, P. Foris, A. I. Frenkel, A. L. Ankudinov, J. Tominaga, and T. Uruga, Understanding the phase-change 
mechanism of rewritable optical media, Nat. Mater. 3, 703 (2004).

[10] J. Vinson, J. J. Rehr, J. J. Kas, and E. L. Shirley, Bethe-Salpeter equation calculations of core excitation spectra, Phys. Rev. B 83, 115106 (2011).

[11] D. Prendergast and G. Galli, X-Ray Absorption Spectra of Water from First Principles Calculations, Phys. Rev. Lett. 96, 215502 (2006).

[12] F. Ráksi, K. R. Wilson, Z. Jiang, A. Ikhlef, C. Y. Cóté, and J.-C. Kieffer, Ultrafast X-ray absorption probing of a chemical reaction, J. Chem. Phys. 104, 6066 (1996).

[13] H. Nakano, Y. Goto, P. Lu, T. Nishikawa, and N. Uesugi, Time-resolved soft $\mathrm{x}$-ray absorption spectroscopy of silicon using femtosecond laser plasma x rays, Appl. Phys. Lett. 75, 2350 (1999).

[14] C. Bressler, C. Milne, V.-T. Pham, A. ElNahhas, R. M. van der Veen, W. Gawelda, S. Johnson, P. Beaud, D. Grolimund, M. Kaiser, C. N. Borca, G. Ingold, R. Abela, and M. Chergui, Femtosecond XANES study of the light-induced spin crossover dynamics in an iron II complex, Science 323, 489 (2009).

[15] H. T. Lemke et al., Femtosecond x-ray absorption spectroscopy at a hard x-ray free electron laser: Application to spin crossover dynamics, J. Phys. Chem. A 117, 735 (2013).

[16] A. Cannizzo, C. J. Milne, C. Consani, W. Gawelda, C. Bressler, F. van Mourik, and M. Chergui, Light-induced spin crossover in Fe(II)-based complexes: The full photocycle unraveled by ultrafast optical and X-ray spectroscopies, Coord. Chem. Rev. 254, 2677 (2010).

[17] M. Cammarata, R. Bertoni, M. Lorenc, H. Cailleau, S. DiMatteo, C. Mauriac, S. F. Matar, H. Lemke, M. Chollet, S. Ravy, C. Laulhé, J. F. Létard, and E. Collet, Sequential Activation of Molecular Breathing and Bending During Spin-Crossover Photoswitching Revealed by Femtosecond Optical and X-Ray Absorption Spectroscopy, Phys. Rev. Lett. 113, 227402 (2014).

[18] C. Ott et al., Lorentz meets Fano in spectral line shapes: A universal phase and its laser control, Science 340, 716 (2013).

[19] M. Schultze et al., Attosecond band-gap dynamics in silicon, Science 346, 1348 (2014).
[20] A. Cavallieri, M. Rini, H. H. W. Chong, S. Fourmaux, T. E. Glover, P. A. Heimann, J. C. Kieffer, and R. W. Schoenlein, Band-Selective Measurements of Electron Dynamics in $\mathrm{VO}_{2}$ using Femtosecond Near-Edge X-Ray Absorption, Phys. Rev. Lett. 95, 067405 (2005).

[21] Z.-H. Loh and S. R. Leone, Capturing ultrafast quantum dynamics with femtosecond and attosecond x-ray corelevel absorption spectroscopy, J. Phys. Chem. Lett. 4, 292 (2012).

[22] E. R. Hosler and S. R. Leone, Characterization of vibrational wave packets by core-level high-harmonic transient absorption spectroscopy, Phys. Rev. A 88, 023420 (2013).

[23] E. Goulielmakis et al., Real-time observation of valence electron motion, Nature (London) 466, 739 (2010).

[24] J. Stingl, F. Zamponi, B. Freyer, M. Woerner, T. Elsaesser, and A. Borgschulte, Electron Transfer in a Virtual Quantum State of $\mathrm{LiBH}_{4}$ Induced by Strong Optical Fields and Mapped by Femtosecond X-Ray Diffraction, Phys. Rev. Lett. 109, 147402 (2012).

[25] B. Schütte, P. Weber, K. Kovacs, E. Balogh, B. Major, V. Tosa, S. Han, M. J. J. Vrakking, K. Varju, and A. Rouzee, Bright attosecond soft $\mathrm{x}$-ray pulse trains by transient phase-matching in two-color high-order harmonic generation, Opt. Express 23, 33947 (2015).

[26] See Supplemental Material at http://link.aps.org/supplemental/ 10.1103/PhysRevB.95.081101 for experimental methods, data analysis, Raman measurements, and theory.

[27] M. Matsuo and S.-I. Orimo, Lithium fast-ionic conduction in complex hydrides: Review and prospects, Adv. Energy Mater. 1, 161 (2011).

[28] L. Dhar, J. A. Rogers, and K. A. Nelson, Time-resolved vibrational spectroscopy in the impulsive limit, Chem. Rev. 94, 157 (1994).

[29] M. Bargheer, N. Zhavoronkov, Y. Gritsai, J. C. Woo, D. S. Kim, M. Woerner, and T. Elsaesser, Coherent atomic motions in a nanostucture studied by femtosecond $\mathrm{x}$-ray diffraction, Science 306, 1771 (2004). 